\title{
Development and evaluation of a real-time PCR assay for detection of Pneumocystis jirovecii DNA in bronchoalveolar lavage fluid of HIV-infected patients
}

\author{
J F Huggett, ${ }^{1}$ M S Taylor, ${ }^{2}$ G Kocjan, ${ }^{3}$ H E Evans, ${ }^{4}$ S Morris-Jones, ${ }^{5}$ V Gant, ${ }^{5}$ T Novak, \\ A M Costello, ${ }^{6}$ A Zumla, ${ }^{1}$ R F Miller ${ }^{4,7}$
}

${ }^{1}$ Centre for Infectious Diseases and International Health,

Windeyer Institute for Medical Sciences, University College

London, London, UK; ${ }^{2}$ Welcome Trust Centre for Human

Genetics, University of Oxford, Oxford, UK; ${ }^{3}$ Department of Histopathology, University College London, London, UK;

${ }^{4}$ Centre for Sexual Health and HIV Research, Department of Primary Care and Population Sciences, University College London, London, UK;

${ }^{5}$ Department of Microbiology, University College London Hospitals NHS Foundation Trust, Windeyer Institute for Medical Sciences, London, UK:

${ }^{6}$ International Perinatal Care Unit, Institute of Child Health, University College London, London, UK; ${ }^{7}$ Department of Infectious and Tropical Diseases, London School of Hygiene and Tropical Medicine, London, UK

Correspondence to:

Professor R F Miller, Centre for Sexual Health and HIV Research, Department of Primary Care and Population Sciences, University College London, London WC1E 6JB, UK; rmiller@gum.ucl.ac.uk

GeneBank: Accession number D0987621.

Received 22 March 2007 Accepted 27 July 2007

Published Online First

10 August 2007

\section{ABSTRACT}

Background: Pneumocystis pneumonia (PCP) is conventionally diagnosed by identifying Pneumocystis jirovecii in lower respiratory tract samples using cytochemical stains. Molecular diagnosis of PCP is potentially more sensitive.

Methods: A study was undertaken to use an extensively optimised real-time polymerase chain reaction (PCR) using primers designed to hybridise with the $P$ jirovecii heat shock protein 70 (HSP70) gene to quantify $P$ jirovecii DNA in bronchoalveolar lavage (BAL) fluid from HIV-infected patients with and without PCP, and to compare this assay with conventional PCR targeting the $P$ jirovecii mitochondrial large subunit rRNA gene sequence (mt LSU rRNA).

Results: Sixty-one patients had 62 episodes of PCP (defined by detection of $P$ jirovecii in BAL fluid by cytochemical stains and typical clinical presentation). Quantifiable HSP70 DNA was detected in 61/62 (range 〜13-18 608 copies/reaction; median 332) and was detectable but below the limit of quantification $1 \sim 5$ copies/reaction) in $1 / 62$. Seventy-one other patients had 74 episodes with alternative diagnoses. Quantifiable HSP70 DNA was detectable in 6/74 (8\%) episodes (range $\sim 6-590$ copies/reaction; median $\sim 14$ ) and detectable but below the limit of quantification in 34/74 (46\%). Receiver-operator curve analysis (cut-off $>10$ copies/ reaction) showed a clinical sensitivity of $98 \%$ (95\% 91\% to $100 \%)$ and specificity of $96 \%(95 \% \mathrm{Cl} 87 \%$ to $99 \%)$ for diagnosis of PCP. By contrast, clinical sensitivity of $\mathrm{mt}$ LSU rRNA PCR was $97 \%$ (95\% Cl $89 \%$ to $99 \%$ ) and specificity was $68 \%$ (95\% Cl $56 \%$ to $78 \%$ ).

Conclusion: The HSP70 real-time PCR assay detects $P$ jirovecii DNA in BAL fluid and may have a diagnostic application. Quantification of $P$ jirovecii DNA by real-time PCR may also discriminate between colonisation with $P$ jirovecii and infection.

The fungal pathogen Pneumocystis jirovecii is the cause of Pneumocystis pneumonia (PCP) in humans. ${ }^{1}$ Diagnosis of $P$ jirovecii infection is hampered by the lack of a sustainable in vitro culture method. Diagnosis of PCP is typically made by direct examination of respiratory samples (bronchoalveolar lavage (BAL) fluid or induced sputum) after staining in order to detect the cyst form of Pneumocystis. ${ }^{2}$ Some laboratories also use immunofluorescence stains to enhance sensitivity. The use of cytochemical stains for diagnosis is time consuming, and it may be difficult to maintain laboratory diagnostic expertise because of the lower incidence of PCP since the introduction of highly active antiretroviral therapy.

Molecular diagnostic techniques using the polymerase chain reaction (PCR) are more clinically sensitive than staining for detection of $P$ jirovecii in BAL fluid and induced sputum..$^{3-6}$ Molecular techniques, however, may identify $P$ jirovecii DNA in respiratory samples from patients without clinically apparent PCP, ${ }^{7-10}$ suggesting asymptomatic carriage or "colonisation". Several studies have used real-time PCR for detection of $P$ carinii, ${ }^{11}$ $P$ murina ${ }^{12}$ and $P$ jirovecii in respiratory specimens. ${ }^{13-}$ 21

Real-time PCR allows accurate quantification of DNA and the potential to discriminate between asymptomatic carriage of $P$ jirovecii and clinical disease based on pathogen load. The objective of this study was to compare real-time PCR using primers designed to hybridise to the heat shock protein 70 (HSP70) gene of $P$ jirovecii ${ }^{22}{ }^{23}$ with conventional PCR using primers designed to the large subunit of mitochondrial rRNA ( $\mathrm{mt}$ LSU rRNA) ${ }^{3}{ }^{10}$ for detection of $P$ jirovecii DNA in BAL fluid from patients undergoing diagnostic bronchoscopy. Patients had either proven PCP or confirmed alternative diagnoses.

\section{METHODS}

\section{Selection of a new molecular target}

The target region of HSP70 was obtained for $P$ jirovecii by designing generic primers to the flanking regions of $P$ carinii. Nucleotide sequences for all Pneumocystis types available from public data repositories were searched using Blast $\mathrm{X}^{24}$ against the non-redundant protein database. Expressed sequence tag data were assembled into contigs using CAP3 prior to searching in order to remove redundancy and improve sequence accuracy. Sequences with high quality matches (bit score $>60$ ) to proteins from a broad spectrum of eukaryotic species were considered further (minimally: Caenorhabditis elegans, Drosophila melanogaster, Saccharomyces cerevisiae, Schizosaccharomyces pombe and Neurospora crassa).

The corresponding region was amplified using $P$ jirovecii as template and sequenced. Real-time PCR for $P$ jirovecii HSP70 ( $P j \mathrm{HSP70a)}$ was designed to amplify a 106 base pair region of the $P$ jirovecii sequence and not the corresponding sequence of other Pneumocystis species, or a wide range of potential fungal, bacterial or mycobacterial pathogens (table 1). DNA was extracted from these isolates using the DNeasy tissue kit (OIAGEN, 
Table 1 Fungal, bacterial and mycobacterial organisms used for cross-reactivity assessment of the specificity of the PjHSP70a PCR assay

\begin{tabular}{ll}
\hline & Organism \\
\hline Fungi & Aspergillus flavus \\
& Aspergillus fumigatus \\
& Aspergillus niger \\
& Aspergillus terreus \\
& Candida glabrata \\
& Candida kruseii \\
& Candida parapsilosis \\
& Candida tropicalis \\
& Cryptococcus sp \\
& Pneumocystis carinii and Pneumocystis \\
& wakefieldiae \\
& Pneumocystis jirovecii \\
& Pneumocystis murina \\
& Saccharomyces cerevisiae \\
& Trichosporon sp \\
& Burkholderia cepacia \\
& Haemophilus influenzae \\
Bacteria & Propionibacterium sp \\
& Staphylococcus aureus \\
& Streptococcus agalactiae \\
& Streptococcus mitis \\
& Streptococcus oralis \\
& Streptococcus pneumoniae \\
Mammalia & Mycobacterium avium \\
& Mycobacterium tuberculosis \\
& Human DNA \\
\hline &
\end{tabular}

Crawley, UK) following the manufacturer's instructions, and was used as template for the PjHSP70a.

\section{Patients and samples}

One hundred and thirty-six BAL samples were obtained from 132 adult HIV-infected patients (112 men) undergoing diagnostic bronchoscopy. Sixty-one consecutive patients with PCP had 62 episodes of PCP; one patient had two episodes (interval 9 weeks). All had typical clinical and radiographic presentations, identification of $P$ jirovecii cysts in BAL fluid by Grocott-Gomori methenamine silver staining ${ }^{2}$ and response to specific anti-PCP therapy. ${ }^{25}{ }^{26}$ A further 71 consecutive patients (74 episodes) investigated contemporaneously did not have PCP clinically or radiographically, had negative results from Grocott-Gomori staining and did not receive specific anti-Pneumocystis treatment. All had alternative diagnoses, as described previously, ${ }^{10}{ }^{26-28}$ comprising pulmonary Kaposi sarcoma ( $n=21$; one patient had two BAL, interval 27 weeks), bacterial bronchitis $(\mathrm{n}=12)$, lymphocytic interstitial pneumonitis ( $\mathrm{n}=5$; one had two BAL, interval 24 weeks), Cryptococcus neoformans pneumonia $(n=3)$, pulmonary tuberculosis $(\mathrm{n}=3)$, Aspergillus fumigatus bronchitis $(\mathrm{n}=3$; one had two BAL, interval 3 weeks, the diagnosis then was bacterial bronchitis), $S$ pneumoniae pneumonia $(\mathrm{n}=2)$, bronchiectasis $(n=2)$, self-limiting fever and dyspnoea $(n=2)$. One patient each had cytomegalovirus pneumonitis, pulmonary Mycobacterium avium infection, Salmonella typhi septicaemia, tracheitis, adenocarcinoma of bronchus, primary effusion lymphoma, pulmonary Castleman disease and HIV "constitutional" disease. No patient with an alternative diagnosis developed PCP during the 6-month follow-up period.

Outcome (survival at 1 month) was recorded in all patients. Additionally, in those with PCP, receipt of specific antiPneumocystis prophylaxis, disease severity (arterial oxygen

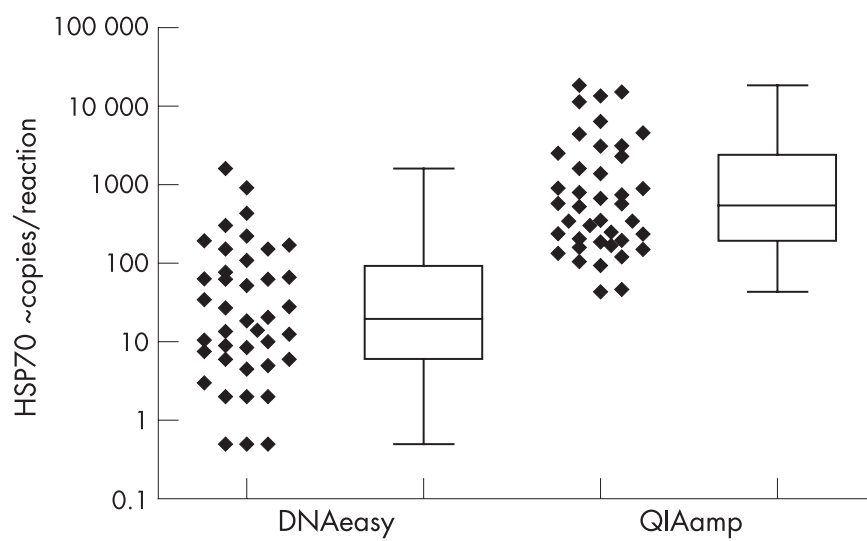

Figure 1 Pneumocystis jirovecii DNA detected in bronchoalveolar lavage fluid from patients with Pneumocystis pneumonia extracted with DNAeasy (left) and QIAamp UltraSens (right). Data are shown as scatter and bar and whisker plots (median, 25th and 75th percentiles and range).

tension $\left(\mathrm{PaO}_{2}\right)$ breathing room air) and number of days of specific anti-Pneumocystis treatment before BAL were also recorded. All bronchoscopies were performed by one of the authors (RFM); BAL was performed in a standardised manner as previously described. ${ }^{25} 26$ All BAL samples were coded and analyses by PCR were performed "blind" to the patient's clinical and laboratory details. All patients undergoing bronchoscopy gave informed written consent and the study was performed with approval from the local research ethics committee.

\section{DNA extraction}

DNA extraction from BAL fluid was first done using the DNeasy tissue kit and subsequently using the OIAamp UltraSens Virus Kit (both OIAGEN, Crawley, UK) following the manufacturer's instructions. Total DNA was extracted from $200 \mu \mathrm{l}$ (DNeasy) or $750 \mu \mathrm{l}$ (OIAamp) BAL fluid. RNA quench was also included in the latter extract, as suggested by the manufacturer, and the extracted sample was eluted in $60 \mu \mathrm{l}$ of elution buffer by contrast with the DNeasy extraction $(100 \mu \mathrm{l}$ of elution buffer). OIAamp UltraSens Virus Kit was chosen as it was specifically designed to extract DNA from liquid samples, enabled a greater volume to be extracted from BAL fluid and facilitated generation of a higher concentration of extracted nucleic acids. Consequently, the final OIAamp eluate achieved a 12.5-fold concentration compared with a twofold increase with the DNeasy kit. When the two extraction techniques were both applied to BAL samples, a greater amount of DNA was extracted with the OIAamp method than with the DNeasy method (fig 1).

\section{Molecular assessment of $\boldsymbol{P}$ jirovecii DNA in BAL fluid}

Attempts were made to use the mt LSU rRNA assay by realtime PCR, but efficient amplification was not possible and so conventional PCR was used for comparison with the PjHSP70a assay.

\section{PjHSP70a}

The $P j \mathrm{HSP} 70 \mathrm{a}$ assay consisted of a $12.5 \mu \mathrm{l}$ reaction using $1 \mathrm{U}$ Hot Taq (BioGene, Kimbolton, UK) with supplied buffer, 3 mM $\mathrm{MgCl}_{2}, 200 \mu \mathrm{M}$ each dNTP, $250 \mu \mathrm{g} / \mu \mathrm{l}$ tRNA (Sigma, UK) and $300 \mathrm{nM}$ of the forward (5'-CGTCTTGTAAACCACTTCATTGC-3') and reverse (5'-AGTCCGTTTAGCACGCTAC-3') 
Table 2 Characteristics of PjHSP70a assay

\begin{tabular}{lllll}
\hline Actual copy number & $\mathbf{5 0 0} \mathbf{0 0 0}$ & $\mathbf{5 0 0}$ & $\mathbf{5 0}$ & $\mathbf{5}$ \\
\hline Average readout $(\mathrm{n}=24$ experiments) & $\sim 597247$ & $\sim 439$ & $\sim 35$ & $\sim 2.26$ \\
Coefficient of variation & $19 \%$ & $24 \%$ & $67 \%$ & $59 \%$
\end{tabular}

primers and $75 \mathrm{nM}$ of the probe (Fam 5'-AAGAAAGATCTITCAGGG-3" BHO1; underlined bases are "locked" nucleic acids). The PjHSP70a real-time PCR assay was performed using the Rotorgene 3000 (Corbett Research, Sydney, Australia) with an initial denaturation of $95^{\circ} \mathrm{C}$ for 8 min followed by 45 cycles of $10 \mathrm{~s}: 95^{\circ} \mathrm{C}, 20 \mathrm{~s}: 72^{\circ} \mathrm{C}$ and $30 \mathrm{~s}: 72^{\circ} \mathrm{C}$. The results were determined by comparing unknowns with a 10-fold dilution series from $\sim 10^{6}$ to $\sim 1$ copy/reaction. Dilution series were made from preparations of pGEM-T easy (Promega, Southampton, UK) plasmid containing the amplicon of interest. The plasmids were linearised prior to repurification using a PCR cleanup kit (Oiagen) and quantified using Pico green reagent (Molecular Probes, Invitrogen, Paisley, UK). Reactions were set up using the CAS liquid-handling robot (Corbett Research) placed in a laminar flow hood to reduce contamination. All clinical samples and standards were run as duplicate PCR reactions.

Negative controls (ultrapure RNAse-free water, Sigma) were distributed to provide maximum information as to the potential source of any contamination. Three negative controls were aliquoted prior to dilution series, three further controls were established before aliquoting clinical samples and an additional four controls were prepared at the end. In summary, 10 negative controls were included in each PCR reaction and a total of 110 negative control PjHSP70a reactions were performed in the assessments.

Reactions were also initially assessed by agarose gel electrophoresis ( $3 \%$ agarose gel with TBE and ethidium bromide) and products were sized using the HYPER ladder IV and V (Bioline, London, UK). The amounts of extracted DNA used for the $P j \mathrm{HSP} 70 \mathrm{a}$ assay were $0.5 \%$ of extract obtained by DNAeasy and $2 \%$ of extract obtained by OIAamp UltraSens. As real-time PCR results are quantitative, a receiver-operator curve (ROC) was generated from the data. No universal reference material was available with which to compare findings, so ROC analysis was used to establish the optimum cut-off which enabled discrimination between PCP and alternative diagnoses.

\section{mt LSU rRNA}

The mt LSU rRNA assay was a $25 \mu \mathrm{l}$ reaction using $1 \mathrm{U}$ Hot Taq (BioGene) with supplied buffer, $3 \mathrm{mM} \mathrm{MgCl}_{2}, 200 \mu \mathrm{M}$ each dNTP, $250 \mu \mathrm{g} / \mu \mathrm{l}$ tRNA (Sigma) and $200 \mathrm{nM}$ of primers pAZ102-H and pAZ102-E (5). The mt LSU rRNA PCR assay was performed using the Palm-Cycler (Corbett Research) with an initial denaturation of $95^{\circ} \mathrm{C}$ for $8 \mathrm{~min}$ followed by 40 cycles of $30 \mathrm{~s}: 95^{\circ} \mathrm{C}, 30 \mathrm{~s}: 62^{\circ} \mathrm{C}$ and $60 \mathrm{~s}: 72^{\circ} \mathrm{C}$. Reactions were assessed by agarose gel electrophoresis ( $1 \%$ agarose gel with TBE and ethidium bromide) and products sized using the $3 \mu \mathrm{l}(8 \mathrm{ng} / \mu \mathrm{l})$ HYPER ladder IV (Bioline).

Samples with PCR product showing band density $>24 \mathrm{ng} /$ 400 base pairs HYPER ladder IV band were scored as positive; samples with no product or product showing the same of less density were scored as negative. Real-time PjHSP70a and conventional mt LSU rRNA PCR data were compared.

\section{Statistical analysis}

For patients with PCP, duration of anti-Pneumocystis treatment before $\mathrm{BAL}, \mathrm{PaO}_{2}$ levels and quantity of $P$ jirovecii DNA were

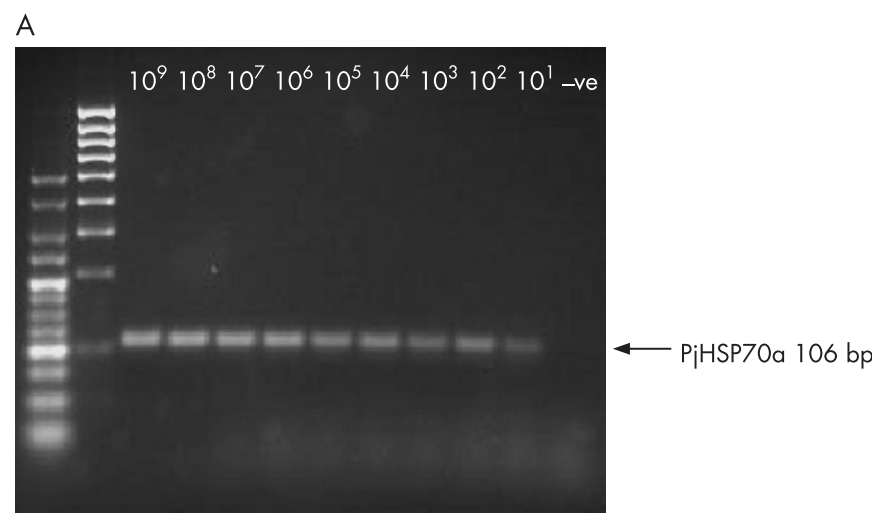

B

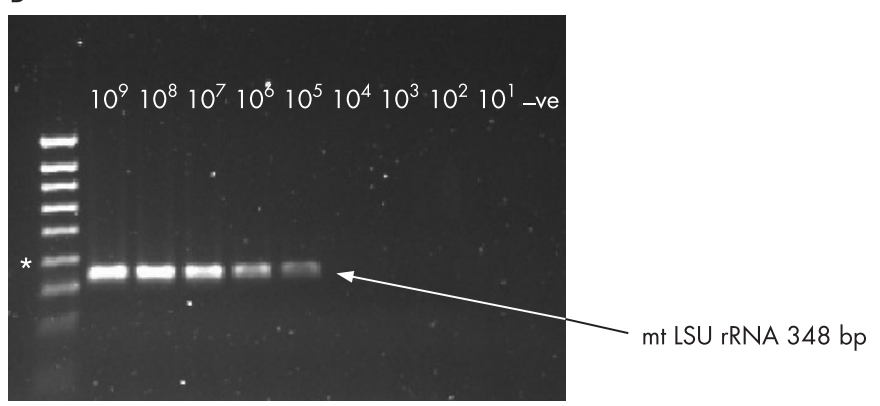

Figure 2 Agarose gel showing analytical sensitivity and dynamic range of PCR assays. (A) PjHSP70 (product, 106 base pairs). (B) mt LSU rRNA (product, 348 base pairs).

$\log _{10}$ transformed to fit a normal distribution. Univariate analyses were performed using Spearman's rank test for continuous variables and the Mann-Whitney test for binary variables to assess correlations between variables and the amount of detectable $P$ jirovecii DNA in BAL fluid samples. STATA Version 9.0 was used for statistical analysis; $p$ values $<0.05$ were regarded as statistically significant.

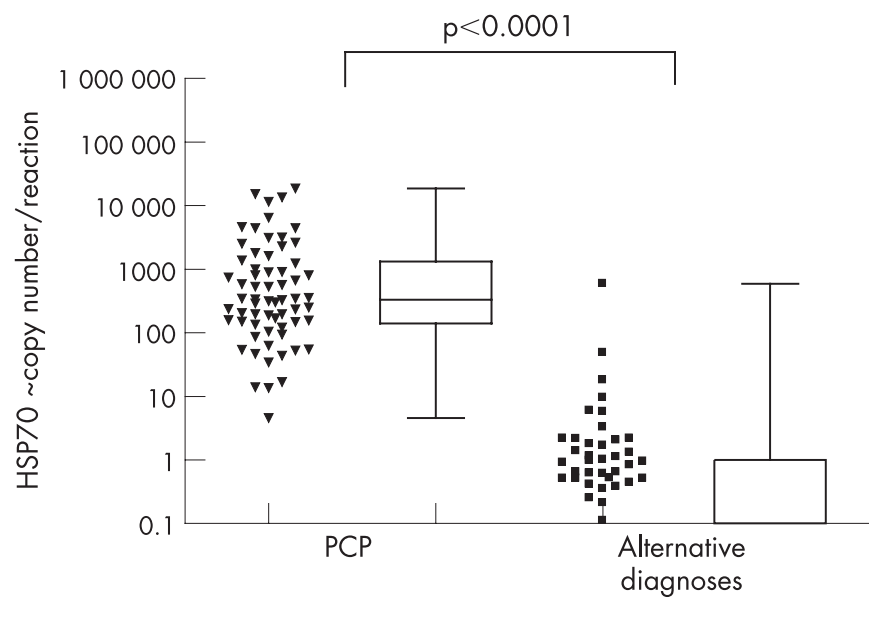

Patients

Figure 3 Real-time PjHSP70a PCR results obtained from OIAamp UltraSens extracted samples from 61 patients with 62 episodes of Pneumocystis pneumonia (PCP, left) and from 71 patients with 74 episodes with alternative diagnoses (right). Data are shown as scatter and bar and whisker plots (median, 25th and 75th percentiles and range). 


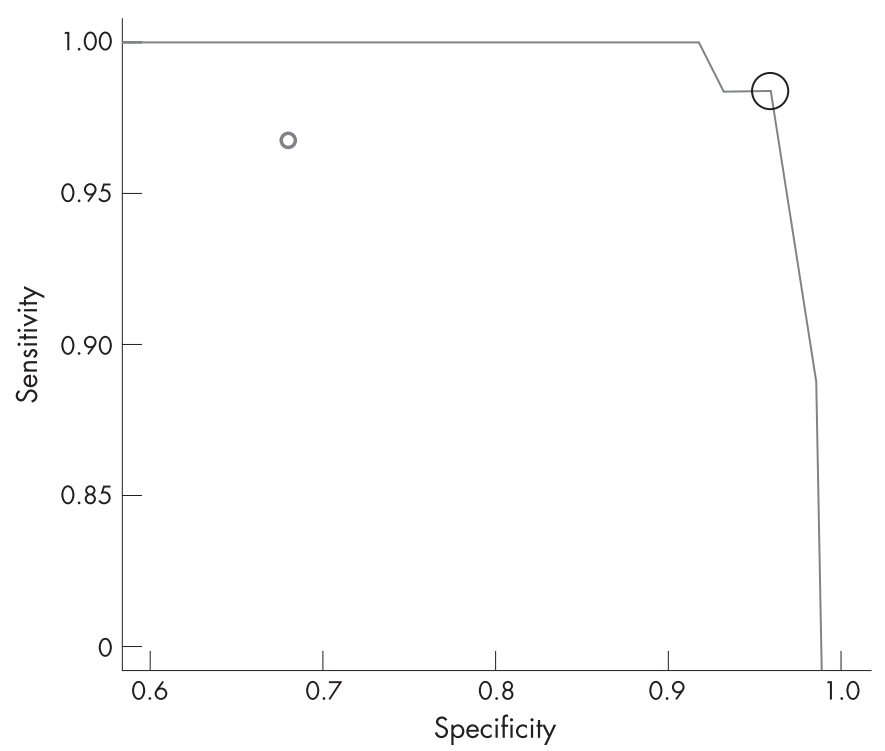

Figure 4 Receiver-operator curve analysis of real-time PjHSP70a PCR assay. The black circle depicts $>10$ copies cut-off used to generate clinical sensitivity and specificity of the PjHSP7Oa assay and the grey circle depicts the sensitivity and specificity of the mt LSU rRNA PCR assay.

\section{RESULTS}

\section{Molecular assay detection sensitivities}

The PjHSP70a assay reproducibly detected as few as $\sim 5$ copies/ reaction (table 2 ) and had a dynamic range across nine orders of magnitude (fig 2A). When the same dilution experiment was performed with the mt LSU rRNA assay, the detection sensitivity was $\sim 10^{5}$ copies/reaction despite extensive optimisation (fig 2B).

\section{Patients}

Among those with PCP, mortality at 1 month was 14.5\% (9/ 62); all patients with alternative diagnoses survived. Among patients with $\mathrm{PCP}, \mathrm{PaO}_{2}$ ranged from 5.1 to $13.2 \mathrm{kPa}$ (median 9.6). Ten patients were receiving prophylaxis. Seven patients had pulmonary co-pathology: pulmonary Kaposi sarcoma $(\mathrm{n}=3), \quad C$ neoformans pneumonia $(\mathrm{n}=2)$, pulmonary Strongyloides stercoralis infection $(n=1)$ and pulmonary tuberculosis $(n=1)$.

\section{Detection of $P$ jirovecii DNA by real-time PCR using the PjHSP70a assay}

In the OIAamp-extracted samples, quantifiable $P$ jirovecii DNA was detectable in 61/62 episodes of PCP (fig 3), ranging from $\sim 13$ to 18608 copies/reaction (median $\sim 332$ ). ROC analysis demonstrated a clinical sensitivity of $98 \%(95 \%$ confidence Interval (CI) $91 \%$ to $100 \%$ ) and specificity of $96 \%$ (95\% CI $87 \%$ to $99 \%$ ) when a quantitative threshold of $>10$ copies/reaction was used to define a diagnosis of PCP (fig 4; sensitivity and specificity were derived from analysis of patients and not total episodes).

One patient with PCP had detectable $P$ jirovecii DNA but below the limits of quantification ( $<5$ copies/reaction). This patient had a first episode PCP, a CD4 count of 0 cells/ $\mu$ l, had not received anti-Pneumocystis prophylaxis and had received 5 days of high-dose co-trimoxazole before BAL was performed. Necropsy confirmed PCP.

Among 71 patients (74 episodes) with an alternative diagnosis, quantifiable ( $>5$ copies/reaction) $P$ jirovecii DNA was detectable in $6(8 \%)$ episodes ( $6-590$ copies/reaction; median 14; table 3), detectable but below the limits of quantification in a further $34(46 \%)$ and was undetectable in 35 (46\%). Levels of detectable quantifiable $P$ jirovecii DNA in those with PCP were higher than in those with alternative diagnoses $(p<0.001$, fig 3 ).

Among patients with PCP there was no correlation between the quantity of detectable $P$ jirovecii DNA and patient's prior receipt of prophylaxis, duration of anti-Pneumocystis treatment before diagnosis, $\mathrm{PaO}_{2}$ or the presence of pulmonary copathology. Of 110 negative controls, only one had detectable DNA ( $<5$ copies/reaction). None of nine other negative controls in that reaction produced a detectable signal.

\section{Detection of $\boldsymbol{P}$ jirovecii DNA using PCR at the mt LSU rRNA}

Pneumocystis jirovecii DNA was detected using the mt LSU rRNA assay in BAL fluid from 61of 62 (96\%) episodes of PCP and from 24 of $74(32 \%)$ episodes with an alternative diagnosis (fig 4). Using the defined cut-off of $>24 \mathrm{ng} /$ reaction gel gave a clinical sensitivity of $97 \%$ (95\% CI $89 \%$ to $99 \%$ ) and a specificity of $68 \%$ (95\% CI $56 \%$ to $78 \%$ ) for a diagnosis of PCP.

\section{DISCUSSION}

This study describes the design, optimisation and performance of a real-time PCR assay intended for detection of $P$ jirovecii. Numerous reports have described molecular assays using conventional PCR to diagnose PCP, the most common being variations on the method targeting $\mathrm{mt}$ LSU rRNA. ${ }^{2}{ }^{10}$ More recently, real-time PCR has been used for the detection of $P$ jirovecii DNA in respiratory samples and for the diagnosis of PCP. ${ }^{13-21} 29$ The present study differs from previous reports by the strategy used for optimising DNA extraction and by the gene target used for analysis. We chose an "in silico" strategy to find an optimal molecular target in the HSP70 gene and compared this method with a well described assay targeting $\mathrm{mt}$ LSU rRNA. ${ }^{2} 310$

Table 3 Patients without Pneumocystis pneumonia who had detectable quantifiable $P$ jirovecii DNA in BAL fluid

\begin{tabular}{llll}
\hline Diagnosis & $\begin{array}{l}\text { CD4 count } \\
\text { (cells/ } \mu \text { I) }\end{array}$ & $\begin{array}{l}\text { Receiving Pneumocystis } \\
\text { prophylaxis (drug) }\end{array}$ & $\begin{array}{l}\text { P jirovecii DNA } \\
\text { ( copies/reaction) }\end{array}$ \\
\hline Aspergillus bronchitis & 30 & Yes (nebulised pentamidine) & 590 \\
Bacterial bronchitis & 20 & No & 49 \\
Pulmonary KS & 190 & Yes (co-trimoxazole) & 18 \\
LIP & 140 & Yes (co-trimoxazole) & 10 \\
Bacterial bronchitis & 200 & No & 6 \\
Bacterial bronchitis & 240 & No & 6 \\
\hline
\end{tabular}

KS, Kaposi sarcoma; LIP, lymphocytic interstitial pneumonitis. 
There are many reasons for selecting this new target: the HSP70 gene is conserved across eukaryotic organisms, ${ }^{22}{ }^{30}$ yet the sequence identity is not. Phylogenetic analysis suggests this sequence is unlikely to be lost, reducing the likelihood of sensitivity being compromised. As this sequence is diverse, the potential for a molecular method detecting other organisms that may be present within the sample-either as a co-pathogen or as a commensal-are greatly reduced. This PCR assay is specific for $P$ jirovecii as it does not detect other species of Pneumocystis, including $P$ carinii, $P$ murina or $P$ wakefieldiae, or a wide range of fungal, bacterial or mycobacterial potential pathogens. Furthermore, by designing the HSP70 assay to detect part of the coding sequence, polymorphisms affecting the resultant heat shock protein are also less likely than if the assay amplified a less conserved non-coding region or a highly variable protein such as the major surface glycoprotein.

When the PjHSP70a assay is used to amplify DNA from BAL fluid, the amounts of $P$ jirovecii HSP70 DNA detected from patients with PCP and those with alternative diagnoses were significantly different. Using a quantitative cut-off of $>10$ copies/reaction, ROC analysis showed clinical sensitivity of $98 \%$ and specificity of $96 \%$. Furthermore, in those with PCP, the amount of $P$ jirovecii DNA in BAL fluid was not associated with disease severity and outcome, suggesting that other factors are implicated in the pathogenesis of PCP.

Interpretation of the significance of finding $P$ jirovecii DNA in a respiratory sample is hampered by the observation that the organism may be asymptomatically present in the lung, either transiently or latently. ${ }^{7-10} 31$ Furthermore, a "negative" result from attempts at DNA detection in the context of asymptomatic carriage becomes more difficult to interpret when using conventional PCR. A faint band following gel electrophoresis might be due to a low starting copy number, which may or may not be attributable to "colonisation"; however, inhibition and/ or low assay efficiency may also contribute to such a finding. There are further difficulties if a mitochondrial sequence such as mt LSU rRNA is used, as little is known about the physiology of $P$ jirovecii and how the mitochondrial DNA copy number (a factor of mitochondrial numeracy and genome replication) may vary according to different states of disease, "colonisation" or in response to treatment.

We found that the mt LSU rRNA PCR assay had an analytical sensitivity for detecting $P$ jirovecii DNA of $\sim 10^{5}$ copies/reaction. This result is surprising and may in part be explained by the secondary structure found in both primers pAZ102- $\mathrm{H}$ and pAZ102-E when analysed using mfold, ${ }^{32}$ and by the $5^{\circ} \mathrm{C}$ difference in melting temperature between these primers identified using the nearest neighbour prediction method. ${ }^{33}$ Both of these factors could affect PCR efficiency at this locus. This finding suggests that mitochondrial sequences can be in great abundance (at least 1000 copies to every one of HSP70) when $P$ jirovecii is present in BAL fluid, as analytical sensitivity was comparable to the single copy PjHSP70a assay. The mt LSU rRNA PCR assay can be nested to improve sensitivity, with a reported detection sensitivity of a single-copy target. ${ }^{34}$ Our data suggest that nested PCR at the mt LSU rRNA locus is unnecessary as, with an optimised extraction procedure, single round PCR at this locus has a sensitivity of $97 \%$. Whichever conventional PCR strategy is chosen, improved analytical sensitivity will be achieved at the cost of reduced specificity, as increasing numbers of patients with low burden asymptomatic "colonisation" are detected. A quantitative method is therefore essential.
Studies which attempt to quantify $P$ jirovecii DNA from BAL fluid and which aim to correlate genome copy numbers with clinical situations (colonisation or PCP) may potentially be confounded by the influence of variable dilution of BAL fluid caused by variations in bronchoscopic technique. In this study we attempted to control for this as one person performed all the BAL using a standard technique. Future studies which control for heterogeneity caused by dilution, either by inclusion of internal amplification controls ${ }^{35}$ or by normalising to genomic DNA, ${ }^{36}$ may enable better clinical correlations to be made.

Several real-time PCR assays using a variety of gene targets have been described for detecting $P$ jirovecii in respiratory samples; ${ }^{12} 1619$ a high inter-laboratory agreement among realtime PCR assays has been described. ${ }^{29}$ Alvarez-Martinez et al compared nested PCR with real-time PCR using the dihydropteroate synthase gene target applied to BAL fluid samples. ${ }^{19}$ Nested PCR had an analytical sensitivity of $94 \%$ and a specificity of $81 \%$ for detection of $P$ jirovecii. Real-time PCR detected $P$ jirovecii DNA from $67 / 70$ patients with microscopically confirmed $\mathrm{PCP}$, range $1-10^{6}$ copies/ $\mu$ l of sample (mean $1.8 \times 10^{4}$ ) and from $3 / 70$ with negative microscopy for PCP, range 1-10 copies/ $\mu$ l of sample (mean 4), giving a clinical sensitivity of $94 \%$ and a specificity of $96 \% .{ }^{19}$ Larsen et al ${ }^{12}$ described development of a quantitative touch-down real-time PCR using the multi-copy major surface glycoprotein (MSG) gene for diagnosis of PCP. Lower respiratory tract samples (BAL fluid and induced sputum) from patients with PCP contained 2.4-1 040000 copies of MSG gene/reaction (median 417) and samples from those without PCP contained 0.3-248 copies/ reaction (median 2.6). Discrimination between infection and colonisation was possible by using an arbitrary cut-off value of 10 copies/reaction. However, applying this cut-off meant that the false positive detection rate fell at the cost of an increase in false negative results. ${ }^{12}$ In a follow-up study the authors applied the MSG real-time PCR assay to oral wash samples for diagnosis of PCP and demonstrated a clinical sensitivity of $88 \%$ and specificity of $85 \% .{ }^{16}$ A marked treatment effect was observed in those with PCP. Samples obtained $\leqslant 1$ day after the start of treatment contained a median of 417 MSG gene copies/reaction (range 0-21 290), and samples from those who had received $>1$ day of treatment contained a median of 7 copies/reaction (range 0-3673). Clinical specificity was increased to $100 \%$ but sensitivity was reduced to $75 \%$ by applying a post hoc cut-off value of 50 copies/reaction. ${ }^{16}$ This observation contrasts with the findings of our study in which we found no correlation between duration of treatment prior to obtaining the BAL fluid sample and HSP70 copy number in those with confirmed PCP. In the present study, if all patients with detectable $P$ jirovecii DNA using the PjHSP70a assay are considered positive, then clinical sensitivity would be $100 \%$ and specificity would be $37 \%$. In order to discriminate between those who did and did not have PCP, we used a cut-off value of $\sim 10$ copies/reaction based on ROC analysis.

Assessments of new molecular diagnostic methods are often reported on a "yes the pathogen is present" versus "no it is not present" basis, depending on whether or not a nucleic acid target is detected in a clinical sample. From such analysis, positive and negative predictive values are derived from what is often a small and highly polarised group of individuals. Diagnostic assays are often not fully characterised, making a negative result difficult to interpret as it is uncertain whether this is a true negative result or is due to sampling procedure, extraction technique or the PCR assay itself. In this study we have demonstrated the importance of DNA extraction 
techniques, as we compared two procedures and showed improved detection of $P$ jirovecii DNA using OIAamp UltraSens. A large number of negative controls were used to exclude potential contamination. Only 1 of 110 negative controls had a detectable signal; this result would have been considered negative using ROC analysis. We interpret this as representing sporadic contamination ( $\sim 1 \%$ of assays).

In summary, molecular detection tests require considerable optimisation in order to be diagnostically useful. In particular, variations in DNA extraction methods may influence the amount of detectable $P$ jirovecii DNA in BAL fluid. Real-time PCR using the HSP70 genomic target enables discrimination of PCP from other infections that form part of the differential diagnosis in immunosuppressed patients. Real-time PCR targeting HSP70 has a potential diagnostic application. A larger prospective translational study is required to define the suitability of this diagnostic approach in a routine clinical setting. The finding of $P$ jirovecii DNA in BAL fluid in some patients without confirmed PCP is consistent with the concept of "colonisation" and underscores the need for a quantitative approach to detection.

Acknowledgements: The authors thank Professor Melanie Cushion, Veterans Affairs Medical Center, Cincinnati, USA for the gift of $P$ carinii, $P$ wakefieldiae and $P$ murina DNA and for insightful advice; and Dr Jeremy Garson, Department of Virology, University College London for helpful discussions on the subject of real-time PCR.

Funding: This research was supported by The Dr Hadwen Trust (JFH, TN, AZ and RFM).

Competing interests: Professor R F Miller is Co-Editor of Sexually Transmitted Infections, part of the BMJ Publishing Group.

\section{REFERENCES}

1. Redhead SA, Cushion MT, Frenkel JK, et al. Pneumocystis and Trypanosoma cruzi: nomenclature and typifications. J Eukaryot Microbiol 2006;53:2-11.

2. Procop GW, Haddad S, Quinn J, et al. Detection of Pneumocystis jiroveci in respiratory specimens by four staining methods. J Clin Microbiol 2004;42:3333-5.

3. Wakefield AE, Pixley FJ, Banerii S, et al. Detection of Pneumocystis carinii with DNA amplification. Lancet 1990;336:451-3.

4. Wakefield $\mathbf{A E}$, Guiver L, Miller RF, et al. DNA amplification on induced sputum samples for diagnosis of Pneumocystis carinii pneumonia. Lancet 1991;337:1378-9.

5. Cartwright CP, Nelson NA, Gill VJ. Development and evaluation of a rapid and simple procedure for detection of Pneumocystis carinii by PCR. J Clin Microbiol 1994;32:1634-8.

6. Caliendo AM, Hewitt PL, Allega JM, et al. Performance of a PCR assay for detection of Pneumocystis carinii from respiratory specimens. J Clin Microbiol 1998;36:97982.

7. Helweg-Larsen J, Jensen JS, et al. Detection of Pneumocystis DNA in samples from patients suspected of bacterial pneumonia: a case-control study. BMC Infect Dis 2002;2:28

8. Maskell NA, Waine DJ, Lindley AL, et al. Asymptomatic carriage of Pneumocystis jiroveci in subjects undergoing bronchoscopy: a prospective evaluation. Thorax 2003:58:594-7.

9. Vargas SL, Ponce CA, Sanchez CA, et al. Pregnancy and asymptomatic carriage of Pneumocystis jiroveci. Emerg Infect Dis 2003;9:605-6.

10. Wakefield AE, Lindley AL, Ambrose HE, et al. Limited asymptomatic carriage of Pneumocystis jiroveci in human immunodeficiency virus-infected patients. $J$ Infect Dis 2003;187:901-8.

11. Larsen HH, Kovacs JA, Stock F, et al. Development of a rapid real-time PCR assay for quantitation of Pneumocystis carinii f. sp. carinii. J Clin Microbiol 2000;40:298993.
12. Vestereng VH, Bishop LR, Hernandez B, et al. Quantitative real-time polymerase chain-reaction assay allows characterization of Pneumocystis infection in immunocompetent mice. J Infect Dis 2004;189:1540-4.

13. Larsen HH, Masur, $\mathrm{H}$, Kovacs JA, et al. Development and evaluation of a quantitative, touch-down, real-time PCR assay for diagnosing Pneumocystis carinii. J Clin Microbiol 2002; 40:490-4.

14. Flori P, Bellete B, Durand F, et al. Comparison between real-time PCR, conventional PCR and different staining techniques for diagnosing Pneumocystis jiroveci pneumonia from bronchoalveolar lavage specimens. J Med Microbiol 2004;53:603-7.

15. Palladino S, Kay I, Fonte R, et al. Use of real-time PCR and the LightCycler system for the rapid detection of Pneumocystis carinii in respiratory specimens. Diagn Microbiol Infect Dis 2001;39:233-6.

16. Larsen $\mathbf{H H}$, Huang $L$, Kovacs JA, et al. A prospective, blinded study of quantitative touch-down polymerase chain reaction using oral-wash samples for diagnosis of Pneumocystis pneumonia in HIV-infected patients. J Infect Dis 2004;189:1679-83.

17. Strutt M, Smith M. Development of a real-time probe-based PCR assay for the diagnosis of Pneumocystis pneumonia. Med Mycol 2005;43:343-7.

18. Arcenas RC, Uhl JR, Buckwalter SP, et al. A real-time polymerase chain reaction assay for detection of Pneumocystis from bronchoalveolar lavage fluid. Diagn Microbiol Infect Dis 2006;54:169-73.

19. Alvarez-Martinez MJ, Miro JM, Valls ME, et al. Sensitivity and specificity of nested and real-time PCR for detection of Pneumocystis jiroveci in clinical specimens. Diagn Microbiol Infect Dis 2006;56:153-60.

20. Kaiser K, Rabodonirina M, Picot S. Real time quantitative PCR and RT-PCR for analysis of Pneumocystis carinii hominis. J Microbiol Methods 2000;45:113-8.

21. Brancart F, Rodriguez-Villalobos H, Fonteyne PA, et al. Quantitative TaqMan PCR for detection of Pneumocystis jiroveci. J Microbiol Methods 2005;61:381-7.

22. Stedman TT, Butler DR, Buck GA. The HSP70 gene family in Pneumocystis carinii: molecular and phylogenetic characterization of cytoplasmic members. J Eukaryot Microbiol 1998;45:589-99.

23. Maher NH, Vermund SH, Welsh DA, et al. Development and characterisation of a molecular viability assay for Pneumocystis carinii $f$ sp hominis. J Infect Dis 2001;183:1825-7.

24. Altschul SF, Madden TL, Schäffer AA, et al. Gapped BLAST and PSI-BLAST: a new generation of protein database search programmes. Nucl Acids Res 1997;25:3389402.

25. Miller RF, Millar AB, Weller IV, et al. Empirical treatment without bronchoscopy for Pneumocystis carinii pneumonia in the acquired immunodeficiency syndrome. Thorax 1989;44:559-64.

26. Miller RF, Kocjan G, Buckland J, et al. Sputum induction for the diagnosis of pulmonary disease in HIV positive patients. $J$ Infect 1991;23:5-15.

27. Miller RF, Loveday C, Holton J, et al. Community-based respiratory viral infections in HIV positive patients with lower respiratory tract disease: a prospective bronchoscopic study. Genitourin Med 1996;72:9-11.

28. Breen RA, Miller RF, Gorsuch T, et al. Virological response to highly active antiretroviral therapy is unaffected by antituberculosis therapy. $J$ Infect Dis 2006; 193:1437-40.

29. Linssen CFM, Jacobs JA, Beckers P, et al. Inter-laboratory comparison of three different real-time PCR assays for the detection of Pneumocystis jiroveci in bronchoalveolar lavage fluid samples. J Med Microbiol 2006;55:1229-35.

30. Karlin S, Brocchieri L. Heat shock protein 70 family: multiple sequence comparisons, function and evolution. J Mol Evol 1998;47:565-77

31. Medrano FJ, Montes-Cano M, Conde $\mathrm{M}$, et al. Pneumocystis jirovecii in general population. Emerg Infect Dis 2005;11:245-50.

32. Zuker M. Mfold web server for nucleic acid folding and hybridization prediction. Nucl Acids Res 2003;31:3406-15.

33. Sugimoto N, Nakano S, Yoneyama M, et al. Improved thermodynamic parameters and helix initiation factor to predict stability of DNA duplexes. Nucl Acids Res 1996;24:4501-5.

34. Nevez G, Magois E, Duwat $H$, et al. Apparent absence of Pneumocystis jirovecii in healthy subjects. Clin Infect Dis 2006;42:e99-101.

35. Hoorfar J, Malorny B, Abdulmawjood A, et al. Practical considerations in design of internal amplification controls for diagnostic PCR assays. J Clin Microbiol 2004;42:1863-8.

36. Hakim H, Gibson C, Pan J, et al. Comparison of various blood compartments and reporting units for the detection and quantification of Epstein-Barr virus (EBV) in peripheral blood. J Clin Microbiol 2007;45:2151-5. 
model does apply to occupational causation overall. However, as they readily acknowledge, an attempt to stratify this relationship according to whether the causative agent is of high molecular weight (HMW) or low molecular weight (LMW) is limited by the small size of the study population. An epidemiological approach offers a means of complementing the observations from this type of clinical study in order to better characterise how the frequency of rhinitis varies with type of exposure.

National reporting schemes for occupational diseases such as The Health and Occupation Reporting (THOR) network ${ }^{2}$ in the UK provide data on the causative agents for a large number of cases of OR and OA reported by networks of respiratory and occupational physicians. In a preliminary study we have determined the number of cases of rhinitis (with or without asthma) as a proportion of the total number of cases of rhinitis and/or asthma for the 15 respiratory sensitisers most frequently reported to THOR during the decade 1997-2006. ${ }^{3}$

The data suggest significant differences in the rhinitis:asthma ratio between the various causative agents. Few, if any, of the cases of OR reported to THOR would have been confirmed by acoustic rhinometry, and their diagnosis is probably based on a history of nasal symptoms, sometimes accompanied by rhinoscopy, which can have poor specificity. This, together with potential reporting biases, limits conclusions that can be drawn from these provisional THOR data. They do, however, raise the possibility that respiratory sensitisers differ in the extent to which they cause rhinitis compared with asthma. This is particularly evident when the rhinitis:asthma ratio is compared for the two most frequently reported respiratory sensitisers-laboratory animals and isocyanates - with a much higher proportion of rhinitis associated with the former.

A number of toxicokinetic factors, such as particle size or solubility, could determine the relative distribution of a sensitiser in the upper and lower airways. Toxicodynamic factors might relate to molecular weight, with the suggestion from the data that higher molecular weight agents might be preferentially associated with rhinitis. The THOR data are consistent with the hypothesis that OR (when in conjunction with OA) is more likely to be caused by sensitisers that cause disease through IgE-mediated mechanisms. On the other hand, LMW agents such as diisocyanates, which may cause OA by non-IgE mechanisms, do not associate strongly with OR. Morphine, which had the highest rhinitis:asthma ratio in our study, may act through direct mast cell degranulation. ${ }^{4}$ Further clinical and epidemiological research could help to substantiate such mechanistic hypotheses. Castano and colleagues have shown that the united airways disease can apply to occupational causation, but this might not be consistent across the diverse range of respiratory sensitisers.

\section{J Seed, M Carder, M Gittins, R M Agius}

Occupational and Environmental Health Research Group, University of Manchester, Manchester, UK

Correspondence to: Dr M J Seed, Occupational and Environmental Health Research Group, Faculty of Medical and Human Sciences, University of Manchester, Oxford
Road, Manchester M13 9PL, UK; martin.seed@ manchester.ac.uk

Competing interests: None.

Accepted 30 March 2009

\section{REFERENCES}

1. Castano D, Gautrin D, Theriault G, et al. Occupational rhinitis in workers investigated for occupational asthma. Thorax 2009;64:50-4.

2. University of Manchester Occupational and Environmental Health. Epidemiology of work related ill health. http://www.medicine.manchester.ac.uk/oeh/ research/workrelatedillhealth/ laccessed 23 Mar 2009).

3. Seed M, Gittins M, De Vocht F, et al. Occupational rhinitis and occupational asthma; one airway two diseases? J Phys: Conf Series 2009;151:012065 http://www.iop.org/EJ/abstract/1742-6596/151/1/ 012065 (accessed 23 Mar 2009)

4. Nasser SMS, Ewan PW. Opiate-sensitivity: clinical characteristics and the role of skin prick testing. Clin Exp Allergy 2001;31:1014-22.

\section{CORRECTION}

doi:10.1136/thx.2007.081687corr1

J F Huggett, M S Taylor, G Kocjan, et al. Development and evaluation of a real-time PCR assay for detection of Pneumocystis jirovecii DNA in bronchoalveolar lavage fluid of HIV-infected patients. Thorax 2008;63: 154-9. There is a typographical error in one of the PCR primers, which will result in the assay not working if this is copied. The forward primer that currently reads 5'-AGTCCGTTTAGCACGCTAC-3' should read 5'-AGTCCGTTTAGCACGCTCAC-3'. 